American Medical Journal 1 (1): 1-7, 2010

ISSN 1949-0070

C 2010 Science Publications

\title{
Hepatotoxicity Due to Histamine Trifluoro-Methyl Toluidide, Amthamine, R-(-)- $\alpha$-Methyl Histamine and Clobenpropit (H1R-H4R-Agonists, Respectively) in Rabbit Experimental Model
}

\author{
${ }^{1}$ Trivendra Tripathi, ${ }^{2}$ Aijaz Ahmed Khan, ${ }^{3}$ Mohammad Shahid, ${ }^{3}$ Haris M. Khan, \\ ${ }^{1}$ Mashiatullah Siddiqui, ${ }^{4}$ Rahat Ali Khan and ${ }^{5}$ Abbas Ali Mahdi \\ ${ }^{1}$ Department of Biochemistry, \\ ${ }^{2}$ Department of Anatomy, \\ ${ }^{3}$ Department of Microbiology, \\ ${ }^{4}$ Department of Pharmacology, \\ Faculty of Medicine, Jawaharlal Nehru Medical College and Hospital, \\ Aligarh Muslim University, Aligarh-202 002, UP, India \\ ${ }^{5}$ Department of Biochemistry, \\ Chhatrapati Shahuji Maharaj Medical University, UP, Lucknow, India
}

\begin{abstract}
Problem statement: Since in vivo studies looking for toxicological impact of histamine receptors-agonists in experimental models are fragmentary, the present study was designed to delineate the histopathological and biochemical changes in livers of rabbits treated with histamine receptors (H1R-H4R)-agonists. Approach: The cohort comprised of six groups (Group I control and Group IIVI treated) containing five rabbits each. Control-group received vehicle (sterile distilled water) and treated groups received subcutaneous histamine $\left(100 \mu \mathrm{g} \mathrm{kg}^{-1} \times\right.$ b.i.d.) and H1R-agonist (HTMT dimaleate), H2R-agonist (amthamine dihydrobromide), H3R-agonist (R-(-)- $\alpha$-methylhistamine dihydrobromide) and H4R-agonist (clobenpropit dihydrobromide) each in a dose of $10 \mu \mathrm{g} \mathrm{kg}^{-1} \times$ b.i.d. Hepatotoxicity due to these agonists analyzed by using histopathological and biochemical methods. Results: Rabbits treated with drugs in group II-VI had significant elevated levels of serum enzymes (alanine aminotransferase (ALT), aspartate aminotransferase (AST), alkaline phosphatase and bilirubin) $(\mathrm{p}<0.05)$. Histopathological examination revealed hepatic congestion (by histamine and H2R-agonist), centrilobular necrosis (H1R-agonist), increasing degree of binuclearity (in H4R-agonist) and rather unusual multinuclearity (in H2R-and H3R-agonist) of hepatocytes and Kupffer cell prominence (in H4R-agonist) constituted the hallmark of the injuries produced by short-term treatment by histamine and its agonists as compared to control group. Conclusion: These results provided evidence that histamine receptors on induction via their specific-agonist produce specific pattern of hepatic congestion, hepatocyte necrosis and polyploidy and Kupffer cell prominence.
\end{abstract}

Key words: Histamine, histamine receptors-agonists, liver histopathology, hepatotoxicity, polypoidy, kupffer cells

\section{INTRODUCTION}

Histamine, a well-known bioactive monoamine, originally considered as a mediator of immediate hypersensitivity, plays an important role in inflammatory and allergic responses. Its biological pleiotropic effects are mediated by four subtypes of histamine receptors (H1R, H2R, H3R and H4R) (Shahid et al., 2009). These receptors have been important drug targets for many years. Their physiological and pathological relevance such as cell proliferation, differentiation, hematopoiesis, embryonic development, regeneration, wound healing, aminergic neurotransmission and numerous brain functions (sleep/nociception, food intake and aggressive behavior), secretion of pituitary hormones, regulation of gastrointestinal and circulatory functions, cardiovascular system (vasodilation and blood pressure reduction), as well as inflammatory reactions, modulation of the immune response, endocrine function

Corresponding Author: M. Shahid, Department of Medical Microbiology, JN Medical College and Hospital, AMU, Aligarh, UP, India Tel: +91-571-2720382 Fax: +91-571-2721776 
and homeostasis are being documented (Shahid et al., 2009). However, the information regarding exact role of Histamine Receptors (HRs) in liver functions and structure is still limited.

As a very active site of metabolism, the liver is especially susceptible to histamine and HRsagonists/antagonists. A recent study showed that the histamine H2R pathway plays a useful role in treatment of endotoxin-induced hepatic injury and related inflammatory disorders (Masaki et al., 2005) and this was also reported earlier that histamine protects against early alcohol-induced liver injury in rats via same pathway (Hornyak et al., 2003). A previous study on $\mathrm{H} 2 \mathrm{R}$-antagonists (cimetidine and ranitidine) has already shown inhibitory effect on liver regeneration, while famotidine (a H2R-antagonist) has been reported not to inhibit liver regeneration (Kanashima and Kobayashi, 1989). Luyendyk et al. (2003) demonstrated that modest inflammation causes the emergence of liver as a target for ranitidine toxicity in rats and suggested a role for inflammation in idiosyncratic reactions to ranitidine. A recent study with impromidine ( $\mathrm{H} 2 \mathrm{R}$-agonist) in rats and dogs demonstrated that impromidine had no serious toxicological effects at lower dose. However, in dogs, at the higher doses minor pathological changes were seen in liver (Lesli et al., 1982).

The data of H1R-, H2R-, H3R- and H4R-receptorsagonists on the effects of hepatic functions and hepatotoxicity are elementary in the existing literature in contrast to studies on their uses in various pathological conditions. This prompted us to look for induction of hepatotoxicity (if any) by H1R-H4Ragonists and we demonstrated here notable hepatotoxicity due to these agonists in albino rabbits by using histopathological and biochemical methods.

\section{MATERIALS AND METHODS}

Experimental design: To evaluate the histopathological changes, New Zealand adult healthy albino rabbits of either sex weighing $1.29 \pm 0.21 \mathrm{~kg}$ were divided into six groups. Each group contained five rabbits.

Control group: Group-I was vehicle (sterile distilled water)-treated.

Experimental group: Group-II histamine-treated; group-III H1R-agonist-treated; group-IV H2R-agonisttreated; group-V H3R-agonist-treated and group-VI was H4R-agonist-treated.

They were housed in well-maintained animal facility at Central Animal House of JN Medical
College, Aligarh Muslim University, Aligarh, in the Bioresources unit under a $12 \mathrm{~h}$ light/dark cycle, temperature $\left(22 \pm 2^{\circ} \mathrm{C}\right)$ and were allowed free access to standard laboratory diet including fresh green vegetables and clean tap water. All studies were carried out during the light cycle and were approved by the Institutional Animal Ethical Committee.

Drugs: Following drugs were used: histamine dihydrochloride from Himedia laboratories Pvt Limited, India; H1R-agonist (Histamine Trifluoro-Methyl Toluidide (HTMT-dimaleate), H2R-agonist (amthamine dihydrobromide), H3R-agonist (R-(-)- $\alpha-$ methylhistamine dihydrobromide) and H4R-agonist (clobenpropit dihydrobromide) which were kindly donated by Tocris Bioscience, Tocris Cookson Ltd., United Kingdom. All doses were referred to the weight of the salts used.

Dose: Histamine dihydrochloride $(100 \mu \mathrm{g} \mathrm{kg}$ ) and other agonists (HTMT-dimaleate, amthamine dihydrobromide, R-(-)- $\alpha$-methylhistamine dihydrobromide, clobenpropit dihydrobromide) (10 $\mu \mathrm{g} \mathrm{kg}^{-1}$ ) were administered twice a day subcutaneously (s.c.) for 10 subsequent days.

\section{Sample collection and analysis methods:}

Blood sample collection and biochemical analysis: To determine biochemical levels for Liver Function Test (LFT), blood samples were collected from rabbits through the marginal ear veins at day 0 prior to drug treatment, as well as on days 3 (the day during drug treatment) and 10 (the day after last day of drug administration). Blood samples were kept at room temperature for $2 \mathrm{~h}$ and then at $4^{\circ} \mathrm{C}$ overnight. Blood samples were centrifuged for $10 \mathrm{~min}$ at $580 \times \mathrm{g}$ and serum was separated and stored in aliquots containing sodium azide as a preservative at $-20^{\circ} \mathrm{C}$ till tested further. Serum levels of liver enzymes, including aspartate aminotransferase (AST) and alanine amino transferase (ALT), alkaline phosphatase and bilirubin, were determined using an automatic analyzer (Transasia XL 300, Gernany).

Liver sample collection and histopathological analysis: For histopathological examination, albino rabbits in each group were sacrificed by high dose ether anesthesia after completion of drug treatments. Animals were immediately perfused with $10 \%$ buffered formalin. Whole liver was taken out and photographed with digital camera (Olympus-SP-560UZ-Indonesia). As 5-1 cm blocks of liver were processed for paraffin embedding. $10 \mu \mathrm{m}$ thick paraffin sections were stained 
with Haematoxylin and eosin and were examined under light microscope (Olympus-BX 40-Japan). Observations were recorded in sample photomicrographs taken at X200, X400 and X1000.

Statistical analysis: Liver Function Tests (LFT) of the experimental rabbits in each group were presented as mean \pm SD. Significance of differences from control values were determined with the paired Student's t test using SPSS 12.0 for windows, Inc., Chicago, IL. In all analysis, $\quad \mathrm{p}<0.05$ was considered as statistically significant.

\section{RESULTS}

To evaluate the toxicological effects of specific histamine receptors-agonists in rabbits both histopathological and biochemical assays were assessed.

Biochemical examination: Rabbits of control group (sterile distilled water-treated) had no significant increase in ALT, AST, alkaline phosphate and bilirubin levels. While rabbits in group II to VI had increased serum levels of these enzymes at days 3- and 10- postdrug treatment compared with control group ( $\mathrm{p}<0.05$ for each drug treated group) (Table 1). However, serum study of day 0 (prior to drug-treatment and vehicletreatment in groups II-VI and group I, respectively) showed no significant changes in the enzyme levels and demonstrated normal condition of liver in all experimental rabbits.

Histopathological examination: On gross examination, the size of the liver of the rabbits from control and experimental group appeared very much alike. In general, the color of liver from control and histamine treated looked dark and alike, those of H1Rand H2R-agonist groups were lighter and those from H3R- and H4R-agonist were paler. Images of liver on zoom revealed some interesting pattern of the hepatic lobules. In all groups except H2R-agonist-treated, they appeared as characteristic regular hexagons. In H2Ragonist group, it was distorted and appeared as cluster of beans. In H4R-agonist group, each hexagon was subdivided by triradiate and cruciform grooves into minor lobules.

Under light microscope, group-I (control-group) showed normal lobular architecture, hepatocyte laminae and patent sinusoids without congestion and hepatocytes appeared normal with only occasional hepatocytes being binucleated (Fig. 1). There was no apparent sign of necrosis, fibrosis or periportal lymphocytic infiltration.

In rabbits of group-II treated with histamine dihydrochloride the contour of hepatocyte laminae and portal tract appeared normal but sinusoids were congested. There was a noticeable increase in the binuclearity of hepatocytes as compared to control group (Fig. 1 and 2). Focal necrosis of hepatic cells was also occasionally noticed. Thus, generalized congestion and focal necrosis and increase incidence of binuclearity as compared to control group were observed (Fig. 1 and 2).

Group-III H1R-agonist (HTMT-dimaleate)-treated rabbits revealed centrilobular necrosis of hepatic cells, moderate increase in the hepatic macrophages. While increased binuclearity of hepatocytes was also noticed in this group as compared to control group (Fig. 1 and 3 ), the sinusoid were less congested as compared to histamine-treated group.

Table 1: Effects of drug-treatments on liver function test (ALT, AST, alkaline phosphatase and bilirubin serum levels)

\begin{tabular}{|c|c|c|c|c|c|c|c|c|c|c|c|c|}
\hline \multirow{2}{*}{$\begin{array}{l}\text { Experimental } \\
\text { groups } \\
(\mathrm{n}=5)\end{array}$} & \multicolumn{3}{|c|}{$\mathrm{ALT}^{\#}\left(\mathrm{IU} \mathrm{L}^{-1}\right)$ (day) } & \multicolumn{3}{|c|}{$\mathrm{AST}^{\#}\left(\mathrm{IU} \mathrm{L}^{-1}\right)$ (day) } & \multicolumn{3}{|c|}{ Alkaline phosphate ${ }^{\#}\left(\mathrm{IU} \mathrm{L}^{-1}\right)$ (day) } & \multicolumn{3}{|c|}{ Bilirubin $^{\#}\left(\mathrm{mg} \mathrm{L}^{-1}\right)$ (day) } \\
\hline & 0 & $3 \mathrm{rd}$ & 10 th & 0 & $3 \mathrm{rd}$ & 10th & 0 & $3 \mathrm{rd}$ & 10th & 0 & $3 \mathrm{rd}$ & 10th \\
\hline $\begin{array}{l}\text { Group I } \\
\text { (Control) }\end{array}$ & $4.90 \pm 0.3$ & $4.97 \pm 0.01$ & $5.00 \pm 0.1$ & $4.25 \pm 0.25$ & $4.27 \pm 0.07$ & $4.38 \pm 0.1$ & $6.92 \pm 0.02$ & $6.90 \pm 0.1$ & $6.99 \pm 0.1$ & $0.089 \pm 0.099$ & $0.090 \pm 0.002$ & $0.087 \pm 0.007$ \\
\hline $\begin{array}{l}\text { Group II } \\
\text { (Histamine } \\
\text {-treated) }\end{array}$ & $4.91 \pm 0.4$ & $" 17.50 \pm 1$ & " $21.50 \pm 0.5$ & $4.24 \pm 0.1$ & ${ }^{\mathrm{T}} 15.30 \pm 0.3$ & 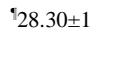 & $6.85 \pm 0.2$ & " $27.20 \pm 0.2$ & " $48.90 \pm 1$ & $0.086 \pm 0.006$ & ${ }^{\mathrm{II}} 0.183 \pm 0.003$ & ${ }^{\mathrm{T}} 0.546 \pm 0.02$ \\
\hline $\begin{array}{l}\text { Group III } \\
\text { (H1R-agonist } \\
\text {-treated) }\end{array}$ & $4.88 \pm 0.08$ & ${ }^{\pi} 34.00 \pm 1.8$ & $" 41.80 \pm 0.8$ & $4.29 \pm 0.2$ & $\mathrm{I}_{34.80 \pm 1}$ & ${ }^{\|} 38.90 \pm 0.2$ & $6.79 \pm 0.4$ & $\mathbb{\pi}_{31.00 \pm 2.5}$ & ${ }^{\mathbb{T}} 38.00 \pm 0.8$ & $0.085 \pm 0.08$ & ${ }^{\mathrm{T}} 0.280 \pm 0.01$ & ${ }^{\mathrm{T}} 0.510 \pm 0.02$ \\
\hline $\begin{array}{l}\text { Group IV } \\
\text { (H2R-agonist } \\
\text {-treated) }\end{array}$ & $4.90 \pm 0.9$ & " $42.70 \pm 2$ & $\mathbb{\pi}_{35.00 \pm 0.5}$ & $4.32 \pm 0.02$ & ${ }^{\mathrm{I}} 34.10 \pm 1$ & ${ }^{\|} 30.20 \pm 0.2$ & $6.88 \pm 0.4$ & $\pi^{\pi} 35.00 \pm 2.5$ & ${ }^{\mathrm{T}} 37.00 \pm 0.8$ & $0.088 \pm 0.008$ & ${ }^{\mathrm{I}} 0.310 \pm 0.01$ & ${ }^{\mathrm{T}} 0.420 \pm 0.02$ \\
\hline $\begin{array}{l}\text { Group V } \\
\text { (H3R-agonist } \\
\text {-treated) }\end{array}$ & $4.93 \pm 0.03$ & ${ }^{\pi} 40.50 \pm 0.5$ & $\pi / 39.80 \pm 1$ & $4.22 \pm 0.1$ & ${ }^{\mathrm{T}} 36.40 \pm 0.4$ & $\pi_{33.80 \pm 3.8}$ & $6.91 \pm 0.2$ & ${ }^{\pi} 34.70 \pm 0.7$ & ${ }^{\mathbb{T}} 39.40 \pm 0.4$ & $0.087 \pm 0.007$ & ${ }^{\mathrm{T}} 0.240 \pm 0.02$ & ${ }^{\mathrm{I}} 0.610 \pm 0.03$ \\
\hline $\begin{array}{l}\text { Group VI } \\
\text { (H4R-agonis } \\
\text {-treated) }\end{array}$ & $4.89 \pm 1$ & $" 40.20 \pm 2$ & ${ }^{\pi} 35.10 \pm 0.1$ & $4.30 \pm 1.1$ & ${ }^{\top} 32.10 \pm 0.1$ & "38.30 \pm 3.3 & $6.86 \pm 0.06$ & " $58 \pm 2.7$ & ${ }^{1} 62.50 \pm 2.5$ & $0.085 \pm 0.005$ & ${ }^{\mathrm{T}} 0.210 \pm 0.02$ & ${ }^{\mathrm{T}} 0.300 \pm 0.05$ \\
\hline
\end{tabular}




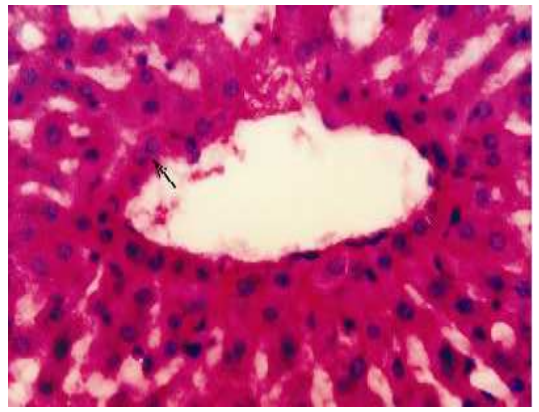

Fig. 1: Control group. In the perivenular region the hepatocytes, cords, sinusoisd normal. Binuclearity $(\uparrow)$ is not very common. X400

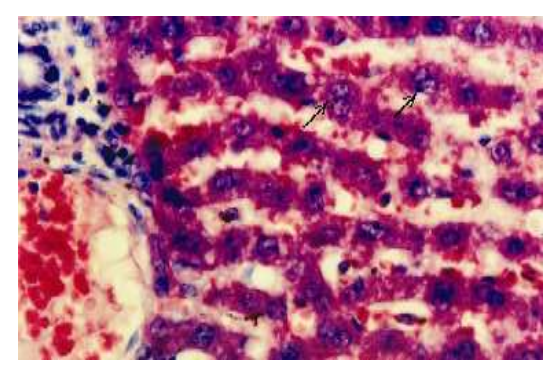

Fig. 2: Histamine-treated group. Architecture is maintained. Portal venule and sinusoids show congestion with mild infiltration in the portal tract. Many binucleated hepatocytes $(\uparrow \uparrow)$ are also seen. X400

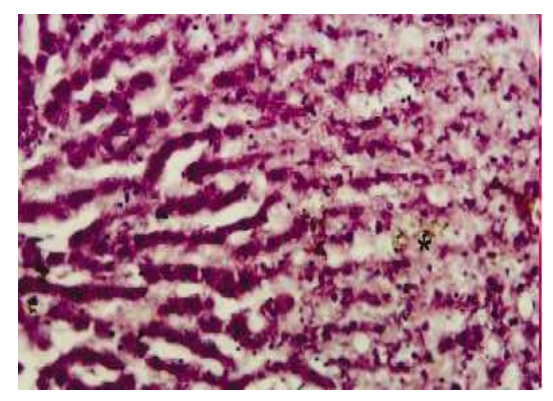

Fig. 3: H1R-agonist-treated group. Centrilobular necrosis seen in the right half of the field with spillover of bile pigment $(*)$. X400

In group-IV H2R-agonist (amthamine dihydrobromide)-treated rabbits, there was marked periportal congestion (Fig. 4) as compared to control as well as with the rest of the groups. Portal tract showed mild infiltration (Fig. 4). Focal necrosis and increased binuclearity were obvious with occasional but unusual polyploidal multinucleated (4 nuclei) hepatocytes very similar to those seen in group- $\mathrm{V}$ were also noticed.

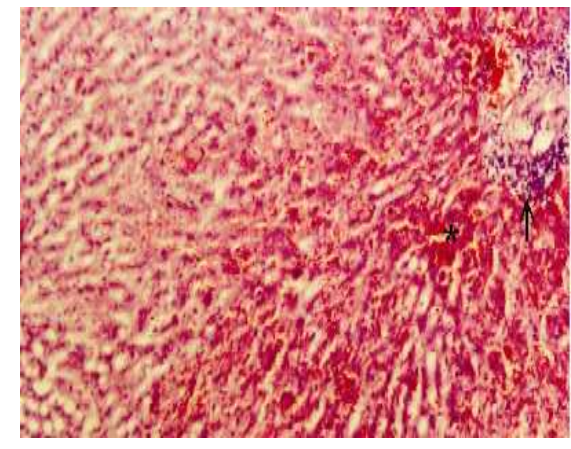

Fig. 4: H2R-agonist-treated group. Marked periportal congestion in the right and lower half of the field $(*)$ with infiltration in the periportal tissue (个). X200

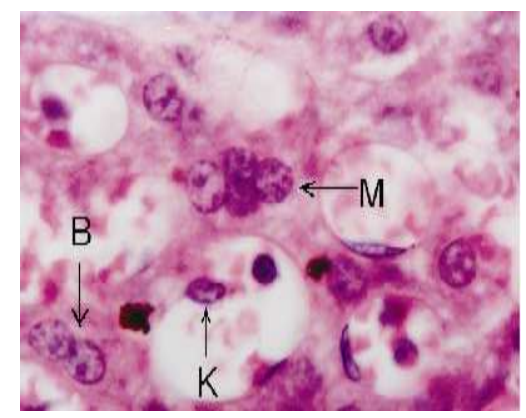

Fig. 5: H3R-agonist-treated group shows Binucleated (B), Multinucleated (M) hepatocytes and Kupffer cell (K). X1000

Group-V-treated with H3R-agonist (R-(-)- $\alpha-$ methylhistamine dihydrobromide) showed focal necrosis, moderate prominence in the Kupffer Cells $(\mathrm{KCs})$ in association with necrotic debris. There was marked nuclear heterogeneity among hepatocytes in terms of their size and number. In addition to increased incidence of binucleated hepatocytes and occasional but rather unusual polyploidal multinucleated hepatocytes were also noticed (Fig. 5), a feature also noticed in group-IV.

Group-VI H4R-agonist (clobenpropit dihydrobromide)-treated rabbits revealed in addition to focal necrosis, marked increased in the binuclearity of hepatocytes $(>60 \%)$ as compared to group-II histaminetreated (Fig. 2 and 6) and other drug-treated groups. One of the remarkable features of this group was prominence of hepatic macrophages or Kupffer Cells (KCs). At certain locations, the hepatocytes were arranged in the form of compact sheet with ill-defined sinusoids warranting further study. 


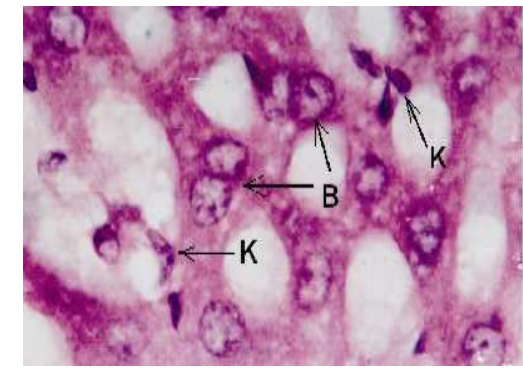

Fig. 6: H4R-agonist-treated group showing Binucleated hepatocytes (B) and Kupffer cells (K). X1000

\section{DISCUSSION}

We demonstrated here that short-term treatment by histamine and histamine receptors-agonists causes increase in the serum levels of ALT, AST, alkaline phosphatase and bilirubin, as well as necrotic changes in hepatocytes, which are biochemical and histological parameters of liver damage, respectively (Thapa and Walia, 2007; Kraly et al., 1996). To provide evidence relating our investigations to in vivo toxicological studies, we used healthy rabbits treated with histamine and histamine receptors (H1R-, H2R-, H3R- and H4R)-agonists. Here we report that histamine and histamine receptors (H1R-, H2R-, H3R- and H4R-)-specific agonist treated rabbits were characterized by marked histological changes as compared to control rabbits. It must be emphasized here that, to the best of our knowledge, none of the earlier reports have demonstrated the biochemical and histopathological studies on H1R-agonist (HTMT-dimaleate), H2Ragonist (amthamine dihydrobromide), H3R-agonist (R$(-)-\alpha$-methylhistamine dihydrobromide) and H4Ragonist (clobenpropit dihydrobromide) which we used in this study.

The elevation of serum levels of ALT, AST, alkaline phosphatase and bilirubin, indicate increased rates of hepatocyte apoptosis and necrosis (Masaki et al., 2005; Thapa and Walia, 2007). Liver apoptosis and necrosis develop in a stepwise fashion. The first step is apoptosis, which is caused by various humoral factors (Masaki et al., 2005; Galanos et al., 1979; Leist et al., 1995; Lehmann et al., 1987). The second step is focal necrosis, which is induced by the accumulated polymorphonuclear cells and lymphomononuclear cells (Galanos et al., 1979; Leist et al., 1995; Lehmann et al., 1987). Finally, massive necrosis appears to be caused by intra-hepatic macrophages and neutrophils (Galanos et al., 1979; Leist et al., 1995; Lehmann et al., 1987). In our study, the liver in the histamine receptorsagonists-treated groups (III-VI) appeared pale in color as compared to the reddish brown color of the controls and histamine treated group. The surface features of hepatic lobules in the zoomed digital images revealed marked distortion (from hexagonal in control) in H4Ragonist treated group possibly due to specific pattern of congestion, infiltration and necrosis. Histological findings in all drug-treated rabbits showed quite specific pattern of degenerative changes in livers. Since histamine is involved in lymphocyte proliferation and neutrophil chemotaxis and infiltration, the possibility exists that histamine prevents liver injury by affecting the late stage of hepatic cell damage, which includes necrosis, induced by inflammatory cells (Masaki et al., 2005). However, the present study demonstrates that short-term treatments of histamine group showed focal necrosis, generalized congestion of sinusoids and marked hepatocyte binuclearity while hepatocyte contour and portal tract appeared normal.

Liver, an organ of vital importance, was markedly damaged by histamine receptors (H1R-, H2R-, H3Rand H4R-)-agonists in the present investigation on rabbits. The damage caused by short-term exposure to histamine and HRs-agonists appeared to be quite specific for each of the agonist. In general, increased incidence of hepatocyte polyploidy (bi-and multinucleated), frequent occurrence of Kupffer Cells $(\mathrm{KCs})$, varied grades of necrosis, congestion and lymphocytic infiltration constituted the hallmark of injuries produced by the histamine and HRs-agonists. Binuclearity of hepatocyte was most remarkable in H4R-agonist treated group while occurrence of multinucleated (4 nuclei) hepatocyte was noticed in H2R- and H3R-agonists treated rabbits. KCs were most pronounced in H4R-agonist treated rabbits. Serum enzymes ALT and AST leakage from hepatocytes and their marked elevations have been shown in cases of hepatitis, neonatal hepatitis, ischaemic and autoimmune toxicity (Thapa and Walia, 2007). AST and ALT levels were significantly increased in all drug-treated groups as compared to control $(\mathrm{p}<0.05)$ and demonstrated the increased rates of liver degeneration in group-II to group-VI with the corresponding values of respective days, which support our histopathological findings in all drug-treated groups compared to control group. Furthermore, increased alkaline phosphatase and bilirubin levels in all drug-treated groups as compared to control group $(\mathrm{p}<0.05)$ also support liver degeneration and sustain our histological findings of liver damage in all the drug-treated experimental groups.

Polyploidy of hepatocytes and prominence of KCs were among the remarkable histopathological changes produced by HRs-agonists. Certain degree of 
binuclearity (about 10\%) and polyploidy in hepatocytes are considered normal (Guidotti et al., 2003). In the present study it has been noticed that histamine and almost all HRs-agonists result into increased incidence of binuclearity (most marked with H4R-agonist) and in certain cases multinuclearity (up to 4 nuclei) of hepatocytes. Though the biological significance of hepatic polyploidy remains unclear, it is believed that binuclear status may constitute an intermediate step before the mononuclear $4 \mathrm{n}$ status is achieved (Guidotti et al., 2003). On one hand polyploidy is considered to be a general physiological process indicating terminal differentiation and senescence or a strategy of cell growth which enables an increase in metabolic output, cell mass, cell size and thus constituting an alternative to cell division. On the other hand, tetraploid to octoploid hepatocytes with one-ortwo nuclei are believe to be due to the cleavage failure (Sigal et al., 1999). Postraumatic hepatic regeneration is associated with cell proliferation, hypertrophy, polyploidization and partial hepatectomy causes accumulation of polyploid hepatocytes with prolonged depletion of diploid hepatocytes (Sigal et al., 1999). It is also believed that polyploidization may affect the expression profile of specific gene and thus provide protection against the dominant expression of mutant onco-genes-a safety measure that might be helpful for an organ heavily engaged in drug detoxification (Sigal et al., 1999). Thus, polyploidization of hepatocyte having single large nucleus or with two nuclei has been described in many conditions but not in association with multinucleated hepatocyte. Therefore, the state of polyploidization of hepatocyte bearing multiple nuclei assumes special significance. In addition, finding of such condition both in H2R- and H3R-agonists treated groups, suggests some kind of functional similarity between the H2R- and H3Ragonists. Therefore, in the present study, it remains to be elucidated as to whether the multinucleated and polyploidal condition of hepatocyte is a regenerative response to necrosis, protective mechanism against oncogenesis or an alternate measure of boosting function during stressful conditions.

Kupffer Cells (KCs) are resident macrophages of the liver. They play an important role in its normal physiology and homeostasis as well as participate in the acute and chronic responses of the liver to toxic compounds (Vbra and Modriansky, 2002). The primary functions of $\mathrm{KCs}$ include phagocytosis, processing of ingested material, antigen presentation and secretion of biologically active products (Vbra and Modriansky, 2002). In the present study, both histamine and most of HRs-agonists treated liver showed mild increase in their incidences. However, H4R-agonist treated liver was characterized by marked increase in the prominence of $\mathrm{KCs}$ in association with pronounced polyploidy (binuclearity) of hepatocytes-feature akin to 'liver cell unrest' which is believed to be common to many serious illnesses affecting the entire body. Erythrophagocytosis by $\mathrm{KCs}$ is said to promote oxidative stress, inflammation and fibrosis (Otogawa et al., 2007). Activation of $\mathrm{KCs}$ by toxic agents results in the release of an array of inflammatory mediators, growth factors and reactive oxygen species (Roberts et al., 2007). Many of the cytokines may induce hepatocyte cell proliferation and may thus enhance clonal expansion of preneoplastic cells leading to neoplasia Roberts et al., 2007). They have an enormous endotoxin eliminating capacity (Prins et al., 2005) and play an important role in both liver damage and regeneration (Baier et al., 2005). They have the same preferences as do spleen cells for certain types of tumor cells. Metastasizing tumor cells are less sensitive than their nonmetastasizing counterparts (Malter et al., 1986). In the present study it remains, to be resolved as to whether the prominence of $\mathrm{KCs}$ in association with marked binuclearity of hepatocytes represents a protective phenomenon against harmful agents or else their idiosyncratic response which might produce undesirable effects.

\section{CONCLUSION}

In summary, the histopathological examination of liver revealed that short-term treatment by histamine and its receptors-agonist produce differential patterns of heptotoxicity in terms of hepatic congestion (histamine and H2R-agonist), centrilobular necrosis (H1Ragonist), binucleated (H4R-agonist) and multinucleated hepatocytes (H2R-and H3R-agonist) and prominent $\mathrm{KCs}$ (H4R-agonist) as compared to control group. In conclusion, these results suggest that histamine receptors on induction via their specific-agonist produce differential pattern of hepatotoxicity warranting further studies.

\section{ACKNOWLEDGMENT}

Trivendra Tripathi acknowledges University Grants Commission, New Delhi, India for providing UGC Fellowship [UGC letter DON F. 19-33/2006 (CU)]. The authors wish to thank Tocris Bioscience, Tocris Cookson Ltd. (United Kingdom) for kindly donating highly potent and specific histamine receptorsagonists and Prof. Abida Malik, Incharge, Central Animal House, J.N.M.C., Aligarh Muslim University, Aligarh for providing animal facilities. 


\section{REFERENCES}

Baier, P.K., U. Baumgartner, S. Hempel, G.W. Vorbeck, E. Von Dobscheutz and U.T. Hopt, 2005. Kupffer cells infiltrate liver tissue early after ischaemiareperfusion and partial hepatectomy. Eur. Surg. Res., 37: 290-297. http://cat.inist.fr/?aModele $=$ afficheN\&cpsidt $=1733$ 7839

Galanos, C., M.A. Freudenberg and W. Reutter, 1979. Galactosamine-induced sensitization to the lethal effects of endotoxin. Proc. Natl. Acad. Sci. USA., 76:

5939-5943.

http://www.pubmedcentral.nih.gov/articlerender.fc gi? artid $=411768$

Guidotti, J.E., O. Bregerie, A. Robert, P. Debey, C. Brechot and C. Desdouets, 2003. Liver cell polyploidization: A pivotal role for binuclear hepatocytes. J. Biol. Chem., 278: 19095-19101. http://www.ncbi.nlm.nih.gov/pubmed/12626502

Hornyak, S.C., K.R. Gehlsen and T. Haaparanta, 2003. Histamine dihydrochloride protects against early alcohol-induced liver injury in a rat model. Inflammation, 27: 317-327. PMID: 14635789

Kanashima, R. and M. Kobayashi, 1989. Famotidine does not inhibit liver regeneration. Eur. Surg. Res., 21: 190-195.

http://cat.inist.fr/?aModele $=$ afficheN\&cpsidt $=6619$ 440

Kraly, F.S., M.E. Keefe, R.A. Tribuzio, Y.M. Kim, J. Finkell and C.V. Braun, 1996. H1, H2 and H3 receptors contribute to drinking elicited by exogenous histamine and eating in rats. Pharmacol. Biochem. Behav., 53: 347-354. http://www.ncbi.nlm.nih.gov/pubmed/8808143

Leist, M., F. Gantner, I. Bohlinger, G. Tiegs, P.G. Germann and A. Wendel, 1995. Tumor necrosis factorinduced hepatocyte apoptosis precedes liver failure in experimental murine shock models. Am. J. Pathol., 146: 1220-1234. http://www.pubmedcentral.nih.gov/articlerender.fc gi?artid=1869293

Lehmann, V., M.A. Freudenberg and C. Galanos, 1987. Lethal toxicity of lipopolysaccharide and tumor necrosis factor in normal and d-galactosaminetreated mice. J. Exp. Med., 165: 657-663. http://jem.rupress.org/cgi/reprint/165/3/657

Lesli, G.B., D.A.A. Owen, F.D. Pollitt, T.J. Sutton and T.F. Walker, 1982. Short-term toxicological studies with impromidine (SK and F 92676): A specific histamine H2-receptor agonist. J. Applied Toxicol., 2: 265-268. PMID: 6223952
Luyendyk, J.P., J.F. Maddox, G.N. Cosma, P.E. Ganey, G.L. Cockerell and R.A. Roth, 2003. Ranitidine treatment during a modest inflammatory response precipitates idiosyncrasy-like liver injury in rats. JPET., $\quad 307: \quad 9-16$. http://www.ncbi.nlm.nih.gov/pubmed/12893837

Malter, M., E. Friedrich and R. Süss, 1986. Liver as tumor cell killing organ: Kupffer cells and natural killers. Cancer Res., 46: 3055-3060. http://www.ncbi.nlm.nih.gov/pubmed/3009008

Masaki, T., S. Chiba, H. Tatsukawa, H. Noguchi and T. Kakuma et al., 2005. The role of histamine H1 receptor and $\mathrm{H} 2$ receptor in LPS-induced liver injury. FASEB. J., 19: 1245-1252. http://www.ncbi.nlm.nih.gov/pubmed/16051691

Otogawa, K., K. Kinoshita, H. Fuji, M. Sakabe and R. Shiga et al., 2007. Erythrophagocytosis by liver macrophages (Kupffer cells) promote oxidative stress, inflammation and fibrosis in a rabbit model of steatohepatitis. Am. J. Pathol., 170: 967-973. http://www.pubmedcentral.nih.gov/articlerender.fc gi? $\operatorname{artid}=1864892$

Prins, H.A., C. Meijer, P.G. Boelens, R.J. Nijveidt and M.P.C. Siroen et al., 2005. The role of Kupffer cells after major liver surgery. J. Parenter. Enteral. Nutr., $\quad 1: \quad 48-55$ http://www.ncbi.nlm.nih.gov/pubmed/15715274

Shahid, M., T. Tripathi, F. Sobia, S. Moin, M.U. Siddiqui and R.A. Khan, 2009. Histamine, histamine receptors and their role in immunomodulation: An updated systematic review. Open Immunol. J., 2: 9-41.

Sigal, S.H., P. Rajvanshi, G.R. Golra, R.P. Sokhi and R. Saxena et al., 1999. Partial hepatectomyinduced polyploidy attenuates hepatocyte replication and activates cell aging events. Am. J. Physiol. Gastrointest. Liver Physiol., 276: G1260G1272. PMID: 10330018

Roberts, R.A., P.E. Ganey, C. Ju, L.M. Kamendulis, I. Rusyn and J.E. Klaunig, 2007. Role of Kupffer cells in mediating hepatic toxicity and carcinogenesis. Toxicol. Sci., 96: 2-15. http://cat.inist.fr/?aModele $=$ afficheN\&cpsidt $=1855$ 8221

Thapa, B.R. and A. Walia, 2007. Liver function tests and their interpretation. Ind. J. Pediatr., 74: 663-671. http://www.ncbi.nlm.nih.gov/pubmed/17699976

Vbra, J. and M. Modriansky, 2002. Oxidative burst of Kupffer cells: Target for liver injury treatment. Biomed. Papers, 146: 15-20. PMID: 12572889 